Acta Crystallographica Section E

Structure Reports

Online

ISSN 1600-5368

Christopher L. Brown, Joel F. Heagney, David J. Young and Peter C. Healy*

School of Science, Griffith University, Nathan, Brisbane 4111, Australia

Correspondence e-mail: p.healy@sct.gu.edu.au

\section{Key indicators}

Single-crystal $\mathrm{X}$-ray study

$T=295 \mathrm{~K}$

Mean $\sigma(\mathrm{C}-\mathrm{C})=0.002 \AA$

$R$ factor $=0.044$

$w R$ factor $=0.140$

Data-to-parameter ratio $=16.2$

For details of how these key indicators were automatically derived from the article, see http://journals.iucr.org/e.

\title{
Methyl 2,5-dihydroxybenzoate
}

The crystal structure of the title compound, $\mathrm{C}_{8} \mathrm{H}_{8} \mathrm{O}_{4}$, is characterized by extensive hydrogen-bonding interactions to yield centrosymmetrically related dimers linked to each other by intermolecular interactions between the hydroxy groups and between the hydroxy and methyl groups.

\section{Comment}

The title compound, (I), was prepared as a prototype receptor with multiple functionality for coordination to analytes. The structure of (I) shows the molecules to be planar (Fig. 1), with bond lengths and angles in accord with conventional values (Allen et al., 1987). Pairs of centrosymmetrically related molecules are associated through bifurcated intra- and intermolecular hydrogen-bonding interactions between the $\alpha$-hydroxy group and carbonyl $\mathrm{O}$ atoms to form a carboxylic acid dimer motif. Further hydrogen-bonding is observed between the 2- and 5-hydroxy groups on adjacent molecules (Fig. 2). In addition, the structure is stabilized through weak $\mathrm{C}-\mathrm{H} \cdots \mathrm{O}$ hydrogen-bonding interactions between the ester methyl group and 5-hydroxy groups on adjacent molecules.<smiles>COC(=O)c1cc(O)ccc1O</smiles>

(I)

\section{Experimental}

2,5-Dihydroxybenzoic acid $(24.6 \mathrm{~g}, 159.5 \mathrm{mmol})$ was dissolved in methanol $(500 \mathrm{ml})$. Sulfuric acid $(98 \%, 10 \mathrm{ml})$ was added dropwise and the mixture stirred under reflux for $72 \mathrm{~h}$. Removal of the solvent under reduced pressure yielded a yellow oil that was partitioned between chloroform $(200 \mathrm{ml})$ and deionized water $(100 \mathrm{ml})$. The organic layer was dried (magnesium sulfate) and condensed to yield the title compound as a crystalline white solid. Yield $24.5 \mathrm{~g}, 80.0 \%$; m.p. 357-359 K (literature: 357-359 K; Malcolm, 1981). Crystals suitable for X-ray diffraction studies were prepared by slow evaporation at room temperature of a solution of $30 \mathrm{mg}$ of (I) in a solution of diethyl ether $(2 \mathrm{ml})$ and hexane $(5 \mathrm{ml})$.

\section{Crystal data}

\section{$\mathrm{C}_{8} \mathrm{H}_{8} \mathrm{O}_{4}$}

$M_{r}=168.14$

Monoclinic, $P 2_{1} / n$

$a=12.316(2) \AA$

$b=12.986(2) \AA$

$c=4.8795(6) \AA$

$\beta=96.406(12)^{\circ}$

$V=775.5(2) \AA^{3}$

$Z=4$
Received 31 March 2003 Accepted 1 April 2003 Online 16 April 2003 
Figure 1

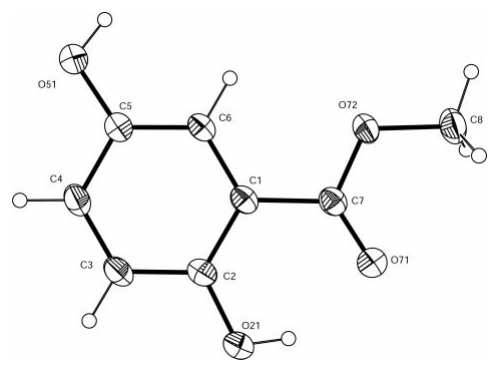

ORTEP-3 (Farrugia, 1997) plot, showing the atomic numbering scheme for (I). Displacement ellipsoids are drawn at the $30 \%$ probability level for non-H atoms.

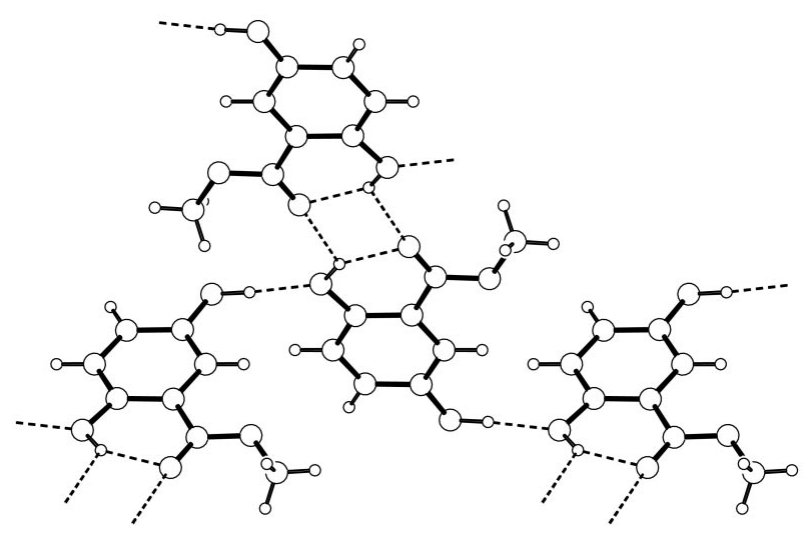

Figure 2

The hydrogen-bonding scheme for (I).

\section{Data collection}

Rigaku AFC-7 $R$ diffractometer $\omega-2 \theta$ scans

Absorption correction: none

2012 measured reflections

1784 independent reflections

1353 reflections with $I>2 \sigma(I)$

$R_{\text {int }}=0.029$

\section{Refinement}

Refinement on $F^{2}$

$R\left[F^{2}>2 \sigma\left(F^{2}\right)\right]=0.044$

$w R\left(F^{2}\right)=0.140$

$S=1.03$

1784 reflections

110 parameters

$\mathrm{H}$-atom parameters not refined $\theta_{\text {max }}=27.5^{\circ}$

$h=-7 \rightarrow 15$

$k=0 \rightarrow 16$

$l=-6 \rightarrow 6$

3 standard reflections every 150 reflections intensity decay: $0.5 \%$

$$
\begin{aligned}
& w=1 /\left[\sigma^{2}\left(F_{o}^{2}\right)+(0.092 P)^{2}\right. \\
& +0.1 P] \\
& \text { where } P=\left(F_{o}^{2}+2 F_{c}^{2}\right) / 3 \\
& (\Delta / \sigma)_{\max }<0.001 \\
& \Delta \rho_{\max }=0.23 \mathrm{e}^{-3} \\
& \Delta \rho_{\min }=-0.19 \mathrm{e}^{-3} \\
& \text { Extinction correction: SHELXL97 } \\
& \text { Extinction coefficient: } 0.033 \text { (9) }
\end{aligned}
$$

\begin{tabular}{|c|c|c|c|c|}
\hline$D-\mathrm{H} \cdots A$ & $D-\mathrm{H}$ & $\mathrm{H} \cdots A$ & $D \cdots A$ & $D-\mathrm{H} \cdots A$ \\
\hline $\mathrm{O} 21-\mathrm{H} 21 \cdots \mathrm{O} 71$ & 0.85 & 1.89 & 2.6205 (17) & 142 \\
\hline $\mathrm{O} 21-\mathrm{H} 21 \cdots \mathrm{O} 71^{\mathrm{i}}$ & 0.85 & 2.43 & $3.0012(18)$ & 125 \\
\hline $\mathrm{O} 51-\mathrm{H} 51 \cdots \mathrm{O} 21^{\mathrm{ii}}$ & 0.85 & 1.92 & $2.7622(17)$ & 169 \\
\hline $\mathrm{C} 8-\mathrm{H} 8 B \cdots \mathrm{O} 51^{\mathrm{iii}}$ & 0.95 & 2.55 & $3.283(2)$ & 134 \\
\hline
\end{tabular}

Table 1

Selected geometric parameters $\left(\AA,^{\circ}\right)$.

\begin{tabular}{llll}
\hline $\mathrm{O} 21-\mathrm{C} 2$ & $1.361(2)$ & $\mathrm{O} 72-\mathrm{C} 7$ & $1.3306(18)$ \\
$\mathrm{O} 51-\mathrm{C} 5$ & $1.363(2)$ & $\mathrm{O} 72-\mathrm{C} 8$ & $1.444(2)$ \\
$\mathrm{O} 71-\mathrm{C} 7$ & $1.2133(18)$ & & \\
$\mathrm{C} 7-\mathrm{O} 72-\mathrm{C} 8$ & $115.78(12)$ & $\mathrm{O} 51-\mathrm{C} 5-\mathrm{C} 6$ & $123.55(13)$ \\
$\mathrm{O} 21-\mathrm{C} 2-\mathrm{C} 3$ & $117.31(13)$ & $\mathrm{O} 72-\mathrm{C} 7-\mathrm{C} 1$ & $113.07(12)$ \\
$\mathrm{O} 21-\mathrm{C} 2-\mathrm{C} 1$ & $123.50(13)$ & $\mathrm{O} 71-\mathrm{C} 7-\mathrm{O} 72$ & $123.09(13)$ \\
$\mathrm{O} 51-\mathrm{C} 5-\mathrm{C} 4$ & $117.16(13)$ & $\mathrm{O} 71-\mathrm{C} 7-\mathrm{C} 1$ & $123.84(14)$ \\
\hline
\end{tabular}

Table 2

Hydrogen-bonding geometry $\left(\AA 0^{\circ}\right)$.

$\mathrm{H}$ atoms were placed at calculated positions with $\mathrm{C}-\mathrm{H}$ set to $0.95 \AA$ A. Hydroxy $\mathrm{H}$ atoms were located from a difference Fourier map and the $\mathrm{O}-\mathrm{H}$ bond length set to $0.85 \AA$. $U_{\text {iso }}$ values for the $\mathrm{H}$ atoms were set at $1.2 U_{\text {eq }}$ of the parent atom.

Data collection: MSC/AFC-7 Diffractometer Control Software for Windows (Molecular Structure Corporation, 1999); cell refinement: MSC/AFC-7 Diffractometer Control Software for Windows; data reduction: TEXSAN for Windows (Molecular Structure Corporation, 1997-2001); program(s) used to solve structure: TEXSAN for Windows; program(s) used to refine structure: TEXSAN for Windows and SHELXL97 (Sheldrick, 1997); molecular graphics: ORTEP-3 (Farrugia, 1997); software used to prepare material for publication: TEXSAN for Windows and PLATON (Spek, 1980-2001).

\section{References}

Allen, F. H., Kennard, O., Watson, D. G., Brammer, L., Orpen, A. G. \& Taylor, R. (1987). J. Chem. Soc. Perkin Trans. 2, pp. S1-19.

Farrugia, L. J. (1997). J. Appl. Cryst. 30, 565.

Malcolm, B. J. (1981). J. Chem. Res. S, p. 193.

Molecular Structure Corporation (1999). MSC/AFC-7 Diffractometer Control Software for Windows. Version 1.02. MSC, 9009 New Trails Drive, The Woodlands, TX 77381, USA.

Molecular Structure Corporation. (1997-2001). TEXSAN for Windows. Version 1.06. MSC, 9009 New Trails Drive, The Woodlands, TX 77381, USA. Sheldrick, G. M. (1997). SHELXL97. University of Göttingen, Germany. Spek, A. L. (1980-2001). PLATON for Windows. Version 121201. University of Utrecht, The Netherlands. 